\title{
Cerium Magnesium Nitrate Temperature Scale from Nuclear Orientation*
}

\author{
R. B. Frankel, $†$ D. A. Shirley, and N. J. Stone \\ Department of Chemistry and Lawrence Radiation Laboratory, \\ University of California, Berkeley, California
}

(Received 21 May 1965)

\begin{abstract}
Systematic deviations were found below $0.003^{\circ} \mathrm{K}$ in the temperature dependence of nuclear orientation of $\mathrm{Ce}^{137 m}$ in cerous magnesium nitrate, using the temperature scale proposed by Daniels and Robinson. The temperature scale below $0.006^{\circ} \mathrm{K}$ was redetermined using a new method: nuclear orientation. This has the advantage over the $\gamma$-ray heating method of high sensitivity at the lowest temperature. The most striking result is that a value of $1 / T$ of 520 , rather than the previously accepted 324 , is obtained by demagnetization from initial conditions of $18.8 \mathrm{kG} \mathrm{deg}^{-1}$. The useful absolute temperature range is thus extended by at least $60 \%$ in $1 / T$. Auxiliary experiments on oriented $\mathrm{Pm}^{144}$ gave similar results and provided independent confirmation both of the inadequacy of the old temperature scale and of the validity of the new one.
\end{abstract}

\section{INTRODUCTION}

$\mathrm{C}$ ERIUM magnesium nitrate $(\mathrm{CMN})$ is unique among paramagnetic working substances in that it may be demagnetized adiabatically from helium bath temperatures $\left(\sim 1^{\circ} \mathrm{K}\right)$ and commonly available magnetic fields of $\sim 20 \mathrm{kG}$ to an absolute temperature at least a factor of three lower than that attainable with the second best pure paramagnetic salt, chromium potassium sulfate. The lowest attainable temperatures to which a specimen may be cooled are thus made available by the use of CMN. ${ }^{1}$ In 1952 Daniels and Robinson (DR) reported ${ }^{2}$ a $T-T^{*}$ correlation for CMN. Here $T^{*}$ is the magnetic temperature, defined from the susceptibility by fitting Curie's law at high temperatures. They discovered the very convenient feature of CMN that $T=T^{*}$ to very low temperatures (within $1 \%$ at $0.006^{\circ} \mathrm{K}$ ). This property has led to the use of $\mathrm{CMN}$ as a thermometer in many experiments in the $0.01^{\circ} \mathrm{K}$ range.

At the lowest temperatures DR found it desirable to employ an "integral heat" method of calorimetry because of the low heat capacity of CMN. This led to a less reliable $T-T^{*}$ correlation at these temperatures. Nonetheless, the DR scale has been in use for 13 years. DeKlerk ${ }^{3}$ reinterpreted the $\mathrm{DR}$ data, concluding that $T=1 / 400$, rather than $T=1 / 324$, was the lowest available temperature. Hudson, Kaeser, and Radford have remeasured the $T-T^{*}$ correlation by similar techniques, finding that the lowest temperature is in the $0.001-0.002^{\circ} \mathrm{K}$ region..$^{4}$

* This work was supported by the U. S. Atomic Energy Commission.

† Present address: National Magnet Laboratory, MIT, Cambridge, Massachusetts.

${ }^{1}$ Of course, the temperature range may be extended down still further by magnetic dilution of CMN or several other salts. For those experiments in which this extension of technique is feasible (dilute) CMN would presumably still be the best working substance.

Lower spin temperatures are attainable by nuclear demagnetization, but until now this has not proved to be a useful cooling technique because the nuclear spins do not achieve equilibrium with the lattice.

${ }^{2}$ J. M Daniels and F. N. H. Robinson, Phil. Mag. 44, 630 (1953).

${ }^{3}$ D. deKlerk, in Handbuch der Physik (Springer-Verlag, Berlin, 1956), Vol. 15, p. 38

${ }^{4}$ R. P. Hudson, R. S. Kaeser, and H. E. Radford, in Proceed
Although CMN has often been used as a thermometer down to $T \cong 1 / 150$, it has been used in its lowest temperature range only for nuclear orientation experiments (including the parity experiment). ${ }^{5}$ In some of these the measured quantities were not temperature-sensitive at the lowest temperatures. In others discrepancies were observed but were not attributed to the DR temperature scale. In two earlier studies in this Laboratory, for example, irregularities in the temperature dependence of $\gamma$-ray angular distributions were noted. ${ }^{6,7}$ With the availability of the new Berkeley 88 in. cyclotron we have been able to restudy the more promising case, $\mathrm{Ce}^{137 m}$, in much greater detail. We have found that the $\mathrm{DR}$ temperature scale for $\mathrm{CMN}$ is very much in error in the lower range, as is deKlerk's modification. In particular, temperatures as low as 1.9 mdeg, rather than $3.1 \mathrm{mdeg}$, are easily reached.

We have constructed a temperature scale based on the nuclear orientation measurements. This is the first temperature scale for a pure paramagnetic salt based on nuclear orientation, and we accordingly discuss this technique in Sec. II. Results are given in Sec. III. The new scale is discussed and related to prospective cooling experiments in Sec. IV.

\section{TEMPERATURE SCALE DETERMINATIONS FOR CMN}

\section{A. Gamma-Ray Heating}

In adiabatic demagnetization experiments it is essential to know the absolute temperature $T$ in terms of easily measurable quantities. One such quantity is the entropy $S$ which is the same after as before demag. netization. The entropy before demagnetization may be directly measured, or, if the partition function of the salt is accurately known, calculated from the initial magnetic field and temperature. To the extent

ings of the VII International Conference on Low Temperature Physics (The University of Toronto Press, Toronto, 1961), p. 100. ${ }^{5}$ C. S. Wu, E. Ambler, R. W. Hayward, D. D. Hoppes, and R. P. Hudson, Phys. Rev. 105, 1413 (1957)

${ }^{6}$ R. W. Grant and D. A. Shirley, Phys. Rev. 130, 1100 (1963). 7 J. N. Haag, D. A. Shirley, and D. H. Templeton, Phys. Rev. 129, 1601 (1963). 
that the lattice entropy is negligible, $S$ is a function of $(H / T)_{i}$. On demagnetization from each value of $(H / T)_{i}$ a magnetic temperature $T^{*}=C / \chi$ is reached. Here $\chi$ is the magnetic susceptibility and $C$ is the Curie law constant, evaluated from the susceptibility at higher temperatures. Since $T^{*}$ is shape-dependent, it is more useful to tabulate $T_{s^{*}}$, the magnetic temperature of a spherical sample. ${ }^{8}$ An absolute temperature also corresponds to each $(H / T)_{i}$, and the relation of these temperatures is called the $T-T_{s}{ }^{*}$ correlation.

In the method of $\gamma$-ray heating, the heat input $Q$ and entropy are correlated by heating the demagnetized sample through absorption of $\gamma$ radiation. The susceptibility is measured and $T_{s}^{*}$ is treated as an independent variable. The temperature is obtained as

$$
T=d Q / d S=\left(d Q / d T^{*}\right) /\left(d S / d T^{*}\right) .
$$

A major weakness of the method is that the data must be differentiated. This is especially harmful at the lowest temperatures.

Another problem that arises in CMN is that $T_{s}{ }^{*}$ becomes an insensitive parameter, varying only slowly with $T$. It is then advisable to use $(H / T)_{i}$ directly as the independent variable, demagnetizing from different fields into the region where $T_{s}{ }^{*}$ does not vary and heating into the sensitive region.

This "integral heat" method has the disadvantage that in heating the specimen through a considerable temperature interval at the lowest temperatures heatleak corrections are particularly difficult to make. Differentiation of the resulting "integral heat" taken as a function of $S$ is thus extremely open to systematic error.

Daniels and Robinson fitted their $Q(S)$ data with a straight line, thus requiring the temperature to be constant for a range of entropy. This is shown to be clearly in error by the nuclear orientation results below. DeKlerk, by neglecting the lowest points, i.e., those with greatest uncertainty, refitted the data, obtaining a different but, as we shall show below, still incorrect temperature scale. The difference of the two scales, and the experimental difficulty of the method, have argued for several years for a redetermination of the CMN temperature scale below $0.006^{\circ} \mathrm{K}$ by a more suitable technique.

Hudson, Kaeser, and Radford ${ }^{4}$ have published preliminary accounts of a redetermination of the temperature scale by essentially the same method. We cannot compare their data in detail with ours as yet, but we note that they also find very low temperatures, in the region 0.001 to $0.002^{\circ} \mathrm{K}$.

\section{B. Nuclear Orientation: A New Method}

Nuclear orientation has been used for thermometry for at least nine years, ${ }^{9}$ but it has not been used before

\footnotetext{
${ }^{8}$ N. Kurti and F. E. Simon, Phil. Mag 26, 849 (1938).

${ }^{9}$ D. F. Griffing and J. C. Wheatley, Phys. Rev. 104, 389 (1956).
}

to determine a temperature scale for a paramagnetic salt. To be applicable this method requires an isotope that goes isomorphously into the lattice, with a wellknown decay scheme, a large $\gamma$-ray anisotropy which does not reach a saturation value in the available temperature range, and a spin Hamiltonian whose form is known. Cerium- $137 \mathrm{~m}$ provides a happy combination of these qualities. The decay scheme is the sequence $11 / 2^{-}(M 4) 3 / 2^{+}$and there are no intermediate states involved. A large anisotropy had been observed in earlier experiments. ${ }^{7}$ The spin Hamiltonian is

$$
\begin{aligned}
\mathfrak{F}=g_{11} \beta H_{z} S_{z}+g_{\perp} \beta\left(H_{x} S_{x}+H_{y} S_{y}\right) & \\
& +A S_{z} I_{z}+B\left(S_{x} I_{x}+S_{y} I_{y}\right),
\end{aligned}
$$

with $B \gg A$. The angular distribution of the $M 4 \gamma$ ray from oriented $\mathrm{Ce}^{137 m}$ in CMN is thus ${ }^{7}$ given by

$$
W(\theta, T)=1-0.889 B_{2} P_{2}(\cos \theta)+0.443 B_{4} P_{4}(\cos \theta) \text {. }
$$

The orientation parameters $B_{2}$ and $B_{4}$ may be calculated from Eq. (2) in the usual way, ${ }^{10}$ in terms of $\beta$ $=B / 2 k T$. By fitting the data to a theoretical curve in the region above $0.006^{\circ} \mathrm{K}$ we derived $B=0.0060 \mathrm{~cm}^{-1}$. As in this region $T=T_{s}^{*}$, we used temperatures calculated from susceptibility measurements using the DR temperature scale. With this value of $B$ the theoretical $W(\theta, T)$ curve is then used to deduce temperatures from gamma-ray ansiotropies observed in the region below $1 / T=150$. As usual the anisotropies observed were corrected for finite detector solid angle and for source decay.

Three separate $\mathrm{Ce}^{137 m}$ experiments were performed using different crystal samples. One of these was spherical; for the other two $T^{*}$ was corrected to $T_{s}{ }^{*}$ using estimated demagnetizing factors. The three sets of data were in excellent agreement.

As a precaution against unknown systematic errors in the $\mathrm{Ce}^{137 m}$ work, additional experiments were performed on $\mathrm{Pm}^{144}$ in CMN. The spin Hamiltonian for $\mathrm{Pm}$ is completely different, being of the form ${ }^{11}$

$$
\mathcal{H C}=P\left[I_{z}{ }^{2}-\frac{1}{3} I(I+1)\right] .
$$

$\mathrm{Pm}^{3+}$ is nonmagnetic and it would not be expected to participate in any possible collective transitions involving the magnetic $\mathrm{Ce}^{3+}$ ions in $\mathrm{CMN}$. The results are discussed in Sec. III and are completely consistent with the temperature scale deduced from the $\mathrm{Ce}^{137 m}$ data.

Nuclear orientation and the older technique have a complementary function in determining an unknown temperature scale. In the higher temperature range the $\gamma$-ray heating method is reliable and, as in this case, is sometimes necessary to make possible measurement of the nuclear orientation parameters. However, as the temperature decreases, systematic errors in the heating

\footnotetext{
${ }^{10}$ R. J. Blin-Stoyle and M. A. Grace, in Handbuch der Physik, edited by S. Flügge (Springer-Verlag, Berlin, 1957), Vol. 42, p. 555 .

${ }^{11}$ C. J. S. Chapman, M. A. Grace, J. M. Gregory, and C. V. Sowter, Proc. Roy. Soc. (London) A259, 377 (1960).
} 


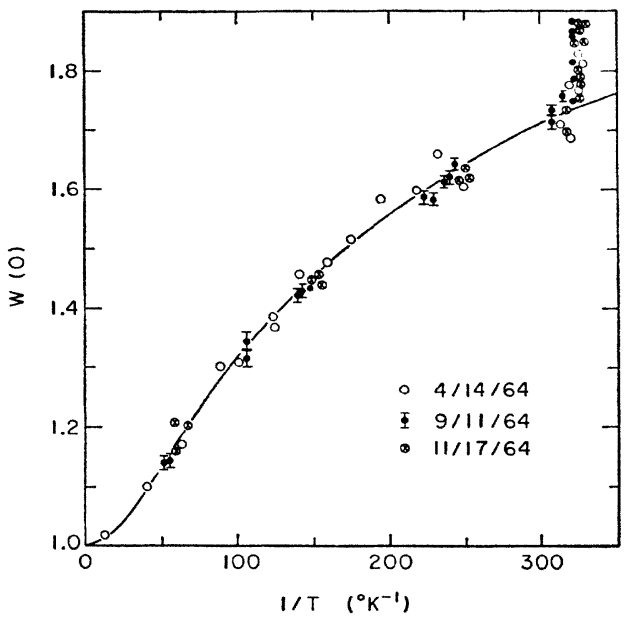

FIG. 1. Normalized intensity along the crystalline $c$ axis of the $255-\mathrm{keV} \gamma$ ray following the decay of $\mathrm{Ce}^{137 m}$ oriented in $\mathrm{CMN}$ versus $1 / T$ on the Daniels and Robinson scale. The theoretical curve was fitted for $T>1 / 150$ by adjusting $B$ in Eq. (2) with $H_{x}=H_{y}=H_{z}=0$. Departure of the data from this curve for $T<1 / 300$ indicates an error in the temperature scale. Different symbols denote different samples.

method become much larger as discussed above, whereas the observed gamma-ray anisotropies increase, making the nuclear orientation technique far more accurate in this region.

\section{RESULTS}

In Fig. 1 we have plotted $W(0)$ for the $255-\mathrm{keV}$ $\gamma$-ray of $\mathrm{Ce}^{137 m}$, oriented in $\mathrm{CMN}$, against $1 / T_{\mathrm{DR}}$, using Daniels and Robinson's temperature scale. The dramatic departure of the data from a fitted theoretical curve at $1 / T_{\mathrm{DR}} \sim 300$ suggests that the temperature scale is in error or that the Hamiltonian suddenly becomes inadequate at this temperature. The latter possibility could be the case if CMN became antiferromagnetic

TABLE I. $1 / \mathrm{T}_{s}{ }^{*}-1 / \mathrm{T}$ correlation for CMN. According to Daniels and Robinson (DR) and this work (FSS).

\begin{tabular}{ccccc}
\hline \hline$(H / T)_{\text {in itial } \mathrm{GG}^{\circ} \mathrm{K}-1}$ & $(S / R)_{\mathrm{cs} \mathbf{l o}}$ & $1 / T_{\mathrm{s}} *$ & $(1 / T)_{\mathrm{DR}}$ & $(1 / T)_{\mathrm{F} \mathrm{SS}}$ \\
\hline 1.0 & 0.691 & 20 & 20 & 20 \\
1.9 & 0.686 & 40 & 40 & 40 \\
2.9 & 0.678 & 60 & 60 & 60 \\
3.8 & 0.667 & 80 & 80 & 80 \\
4.6 & 0.654 & 100 & 100 & 100 \\
5.4 & 0.640 & 120 & 120 & 120 \\
6.2 & 0.625 & 140 & 140 & 140 \\
6.9 & 0.610 & 160 & 160 & 160 \\
7.8 & 0.590 & 180 & 182 & 181 \\
8.75 & 0.567 & 200 & 223 & 210 \\
9.2 & 0.555 & 210 & 231 & 221 \\
9.7 & 0.543 & 220 & 249 & 232 \\
10.2 & 0.529 & 230 & 266 & 249 \\
10.8 & 0.513 & 240 & 284 & 266 \\
11.4 & 0.497 & 250 & 300 & 287 \\
12.1 & 0.477 & 260 & 312.5 & 305 \\
12.8 & 0.459 & 270 & 319 & 322 \\
13.5 & 0.439 & 280 & 322.5 & 358 \\
14.2 & 0.420 & 290 & 324 & 383 \\
15.5 & 0.384 & 300 & 324 & 430 \\
18.0 & 0.321 & 310 & 324 & 500 \\
18.8 & 0.303 & 312 & 324 & 520 \\
\hline \hline
\end{tabular}

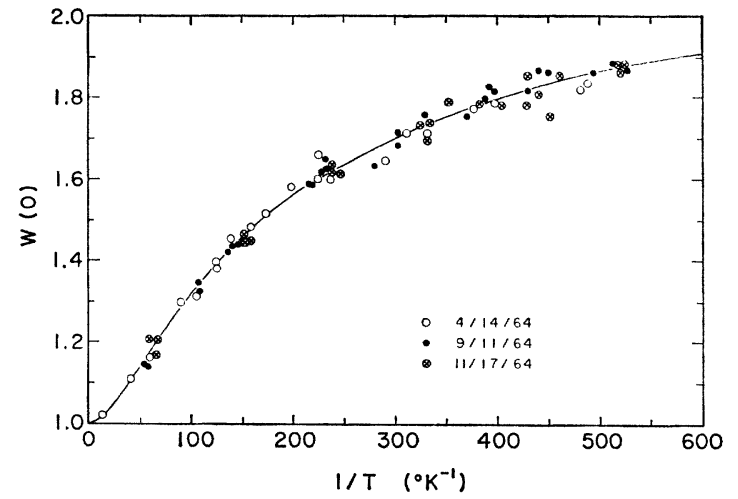

FIG. 2. Normalized intensity along the crystalline $c$ axis of the $\mathrm{Ce}^{137 m} 255-\mathrm{keV} \gamma$-ray versus $1 / T$ using the new temperature scale for CMN. This scale was derived by fitting these data to the theoretical curve.

at $1 / T=300$, for example. However, it is easily shown that antiferromagnetic ordering in the plane perpendicular to the crystalline $c$ axis would lead to a decrease in nuclear orientation, while an increase is observed. An error in the temperature scale is thus indicated.

A new temperature scale was established by fitting the nuclear orientation data for $\mathrm{Ce}^{137 m}$ to the Hamiltonian in Eq. (2) for $T>1 / 150^{\circ} \mathrm{K}$, to determine B, and using this theoretical curve for the lower temperatures. In Table I the resulting temperatures are tabulated against $H_{i} / T_{i}$ and $T_{s}{ }^{*} ; T_{\mathrm{DR}}$ is included for comparison. Figure 2 shows the $\mathrm{Ce}^{137 m}$ data fitted to the theoretical curve for $B=0.0060 \mathrm{~cm}^{-1}$, indicating the lowest temperature reached to be $1 / T=520 \pm 15$. Figure 3 shows our suggested $T-T_{s}^{*}$ relation with the DR scale and deKlerk's version.

It is fortunate that for $\mathrm{Ce}^{137 m}$ the constant coefficient of the $P_{4}(\cos \theta)$ term in $W(\theta, T)$ is large, as below $\sim 0.0022^{\circ} \mathrm{K}$ the $P_{2}(\cos \theta)$ term is close to its maximum value, and the temperature sensitivity relies largely on variation in $B_{4}$. At $1 / T=500$ the coefficient of $P_{4}$ is $+0.215 \pm 0.010$. If, for example, the temperature were really $1 / 700$, this coefficient would be +0.268 .

The relation between $W(0)$ and $W(\pi / 2)$ is sensitive to changes in the nuclear spin Hamiltonian. In Fig. 4

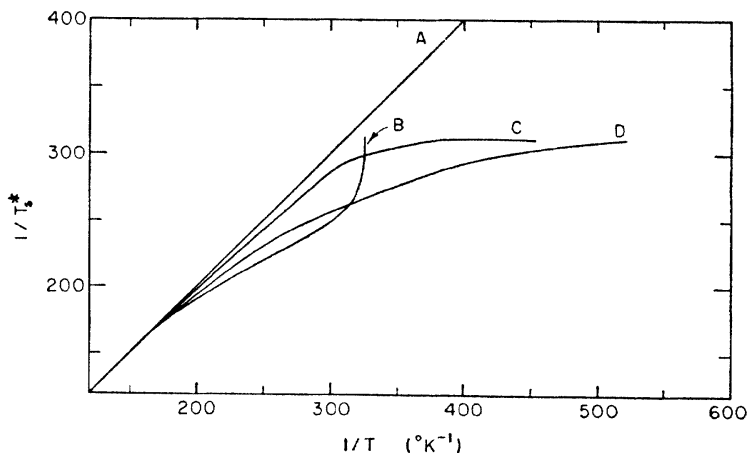

Fig. 3. Comparison of several $T-T_{s} *$ correlations for CMN. Curve A: $T=T_{s}^{*}$. Curve B : Daniels and Robinson. Curve C: deKlerk. Curve D: Present work. The hook in curve B is clearly responsible for the hooks in Figs. 1 and 5. 
FIg. 4. Comparison of normalized tensity, from $\mathrm{Ce}^{137 m}$ oriented in CMN, at 0 and $90^{\circ}$ from the crystalline $c$ axis. Dashed line is for a pure $P_{2}(\cos \theta)$ distribution. 255 -keV $\gamma$-ray in-

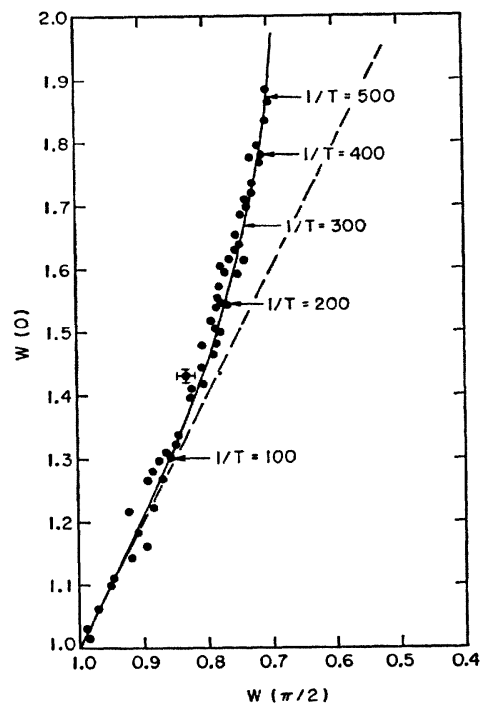

the theoretical curve for pure $M 4$ radiation and planar alignment is compared with the experimental data. The agreement is excellent and in particular no discontinuity is observed in the $1 / T=300$ region.

To obtain a completely independent check of these measurements we aligned $\mathrm{Pm}^{144}$ in CMN, and studied the anisotropies of the 615- and 695-keV gamma rays using $\mathrm{Ge}(\mathrm{Li})$ and $\mathrm{NaI}(\mathrm{Tl})$ detectors. The results are more detailed and accurate than those reported by Grant and Shirley. Again the "hook" in the temperature dependence curve was apparent (Fig. 5) using $T_{\mathrm{DR}}$. However, the new scale allowed a smooth fit (Fig. 6). Although there is considerable uncertainty in the nuclear parameters involved in this decay, ${ }^{6}$ and a detailed discussion would be out of place in this paper, the fact that with physically reasonable parameters a fit is obtained at all temperatures at least shows that the new temperature scale contains no serious

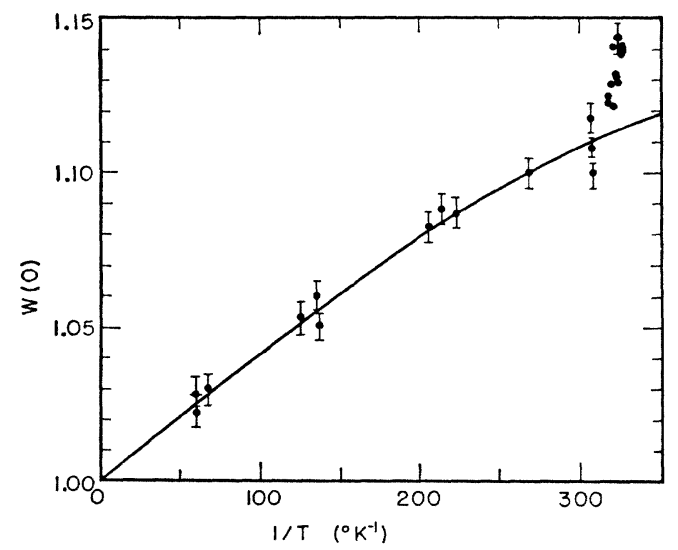

FIG. 5. Temperature dependence of normalized intensity along the crystalline axis of the 615 and $695 \gamma$ rays following the decay of $\mathrm{Pm}^{144}$ oriented versus CMN, using Daniels and Robinson's temperature scale, with a theoretical curve derived from Eq. (4). Again the spurious hook below $T=1 / 300$ is evident.

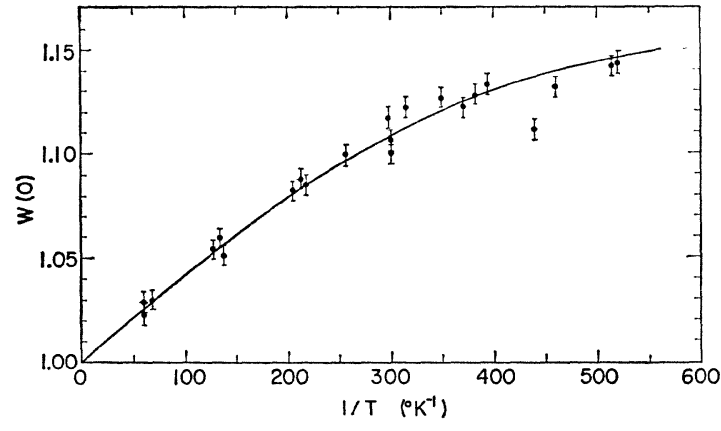

Fig. 6. Normalized gamma-ray intensity data for $\mathrm{Pm}^{144}$, from Fig. 5, plotted against $1 / T$, but using the new $C M N$ temperature scale. Good agreement with the theoretical curve is evident. This serves as an independent check on the new temperature scale.

irregularities. A full analysis of this experiment will be published separately.

\section{DISCUSSION}

The new CMN temperature scale extends the available range of absolute temperature to below $0.002^{\circ} \mathrm{K}$. Considerably lower temperatures may be reached by using larger $(H / T)_{i}$ or by magnetic dilution, and nuclear orientation clearly offers the possibility of determining these temperatures accurately. This temperature region should be useful in connection with searches for very low temperature transitions in superconductors ${ }^{12,13}$ and in $\mathrm{He}^{3}{ }^{34,15}$ It is especially important for the $\mathrm{He}^{3}$ problem that there be no spurious irregularities in the temperature scale. One further aspect of temperature scale determinations should be mentioned. A temperature scale is only useful if it can be reproduced with ease and reliability. It is difficult to grow large clear CMN crystals. The crystals used in this work were not perfectly clear, though they were grown from a solution of many times recrystalized material. This might have an effect on their thermal properties. On the other hand our $T^{*}$ versus $(H / T)_{i}$ data agree well with those of Daniels and Robinson and were very reproducible using different crystals. The scale reported here seems clearly preferable to those previously available, and the usefulness of nuclear orientation in determining temperature scales in this region seems established.

Note added in proof: F. Carboni and R. C. Sapp [Ann. Phys. 33, 77 (1965)] have also found evidence for inadequacy of the DR scale.

\section{ACKNOWLEDGMENT}

We wish to thank Dr. R. P. Hudson for communicating his data prior to publication.

${ }^{12}$ R. A. Hein, J. W. Gibson, R. Mazelsky, R. C. Miller, and J. K. Hulin, Phys. Rev. Letters 12, 230 (1964).

${ }_{13}$ J. F. Schooley, W. R. Hosler, and M. L. Cohen, Phys. Rev. Letters 12, 474 (1964).

${ }^{14}$ V. P. Peshkov, Zh. Eksperim. i Teor. Fiz. 46, 1510 (1964) [English transl.: Soviet Phys.-JETP 19, 1023 (1964)].

${ }_{16}$ W. R. Abel, A. C. Anderson, W. C. Black, and J. C. Wheatley, Phys. Rev. Letters 14, 129 (1965). 